\title{
Erratum: Binary neutron stars with arbitrary spins in numerical relativity [Phys. Rev. D 92, 124012 (2015)]
}

Nick Tacik, Francois Foucart, Harald P. Pfeiffer, Roland Haas, Serguei Ossokine, Jeff Kaplan, Curran Muhlberger, Matt D. Duez, Lawrence E. Kidder, Mark A. Scheel, and Béla Szilágyi

(Received 21 July 2016; published 8 August 2016)

The code used in [Phys. Rev. D 92, 124012 (2015)] erroneously computed the enthalpy at the center of the neutron stars. Upon correcting this error, density oscillations in evolutions of rotating neutron stars are significantly reduced (from $\sim 20 \%$ to $\sim 0.5 \%$ ). Furthermore, it is possible to construct neutron stars with faster rotation rates.

DOI: 10.1103/PhysRevD.94.049903

Our original publication presents a computational code for the construction and evolution of binary neutron stars with arbitrary spin vectors. Following Tichy [1], the 3-velocity of the neutron star fluid in an inspiraling binary is written as the sum of an irrotational part and a rotational part,

$$
U^{i}=\frac{\Psi^{-4} \tilde{\gamma}^{i j}}{h \gamma_{n}}\left(\nabla_{j} \phi+W_{j}\right)
$$

Here $\Psi$ denotes the conformal factor, $\tilde{\gamma}_{i j}$ the conformal spatial metric, $h$ the specific enthalpy, $\gamma_{n}$ the Lorentz term $\gamma_{n}=\left(1-\gamma_{i j} U^{i} U^{j}\right)^{-1 / 2}$, and $\phi$ the irrotational velocity potential. The vector $W_{i}$ represents a rotation term designed to endow a uniform rotation to the star,

$$
W_{i}=\epsilon_{i j k} \omega^{j} r^{k}
$$

where $\omega^{j}$ is the rotation vector chosen by hand, and $r^{k}$ is the distance to the center of the star. In this construction, the solution of the Euler equation is

$$
h=\sqrt{L^{2}-\left(\nabla_{i} \phi+W_{i}\right)\left(\nabla^{i} \phi+W^{i}\right)},
$$

where

$$
\begin{gathered}
L^{2}=\frac{(x+y)+\sqrt{x^{2}+2 x y}}{2 \alpha^{2}}, \\
x=\left(\beta^{i} \nabla_{i} \phi+C\right)^{2},
\end{gathered}
$$

and

$$
y=2 \alpha^{2}\left(\nabla_{i} \phi+W_{i}\right) W^{i} .
$$

Here $C$ denotes the Euler constant, $\alpha$ the lapse function, and $\beta^{i}$ the shift vector.

The code reported in the original article has a mistake in the computation of $h$. Instead of Eq. (3), we computed the following quantity:

$$
h^{\prime}=\sqrt{L^{2}-\left(\nabla_{i} \phi\right)\left(\nabla^{i} \phi\right)},
$$

and instead of Eq. (6), we computed

$$
y^{\prime}=\left(\nabla_{i} \phi+W_{i}\right) W^{i} .
$$




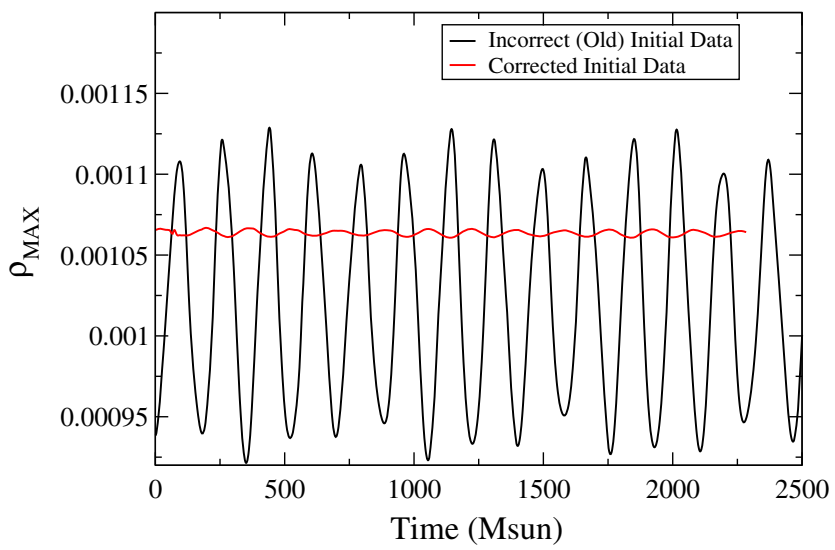

FIG. 1. Density oscillations for the S0.4z run and a new evolution of the corrected initial data.

This error causes $h^{\prime}$ to deviate from the correct $h$ by

$$
h^{\prime 2}-h^{2}=\frac{\left(y^{\prime}-y\right)}{2 \alpha^{2}}+\frac{\sqrt{x^{2}+2 x y^{\prime}}-\sqrt{x^{2}+2 x y}}{2 \alpha^{2}}+W_{i} W^{i}+2 W^{i} \nabla_{i} \phi .
$$

For nonrotating stars, $W^{i}=0$, and the error disappears: $h^{\prime}=h$. In the limit of fast rotation, i.e. large $W$, we expect this difference to be dominated by the terms quadratic in $W$,

$$
h^{\prime 2}-h^{2} \approx \frac{W^{2}}{2 \alpha^{2}} .
$$

This implies the constructed binary neutron star had an enthalpy lower than the correct equilibrium configurations. This picture is consistent with Fig. 20 of the original article (and Fig. 1 below): for high NS spin, the central density $\rho(t)$ immediately increases in an evolution, and oscillates around values larger than the initial density.

\section{UPDATED RESULTS}

We construct initial data with the same input parameters as for the case $50.4 \mathrm{z}-\mathrm{EcC} 3$ and evolve it with the same evolution code. For this evolution, we find

1. Convergence of the Hamiltonian and momentum constraints, and of the ADM energy and ADM angular momentum do not appreciably differ. Convergence of the neutron star spin is somewhat improved.

2. As noted in Ref. [2], the absolute difference between the Komar mass $M_{K}$ and the ADM energy $M_{\mathrm{ADM}}$ is an indicator of deviations from equilibrium, as $M_{K}=M_{\mathrm{ADM}}$ for equilibrium systems in circular orbits. The difference between the Komar mass $M_{K}$ and ADM energy $E_{\mathrm{ADM}}$ is reduced by an order of magnitude, from $\left|M_{K}^{\prime}-M_{\mathrm{ADM}}^{\prime}\right|=2.6 \times 10^{-3}$ to $M_{K}-M_{\mathrm{ADM}}=2.1 \times 10^{-4}$. This supports the idea that the neutron stars themselves are closer to being in equilibrium.

3. Evolution of the corrected initial data yields substantially smaller density oscillations. Figure 1 shows the density oscillations for the evolution reported in the original article and for the evolution of the corrected initial data. Peak-topeak density oscillations are reduced from $\sim 20 \%$ to about $0.5 \%$. Density oscillations of $\sim 0.5 \%$ also occur in our simulations of nonspinning binary neutron stars. The frequency of density oscillation is unchanged, consistent with our interpretation that it represents a quasinormal mode. We note that the phase of oscillation has changed by approximately half of a period.

4. The orbital frequency $\Omega(t)$ has significantly smaller oscillations at periods $\approx 200 M_{\odot}$. Figure 2 compares $\dot{\Omega}(t)$ between evolutions of the old (erroneous) and new (corrected) initial data. High-frequency oscillations are strongly suppressed with the corrected initial data, allowing a clearer view of the lower-frequency sinusoidal features which are due to the overall trajectory of the binary.

5. The corrected code yields higher central density and therefore more compact stars (at the same mass). At the same rotational frequency parameter $\omega$ [as defined in Eq. (2)], we therefore expect the corrected code to yield stars with smaller angular momentum. This is indeed the case, as is shown in Fig. 3. The subsequent evolution of the spin 


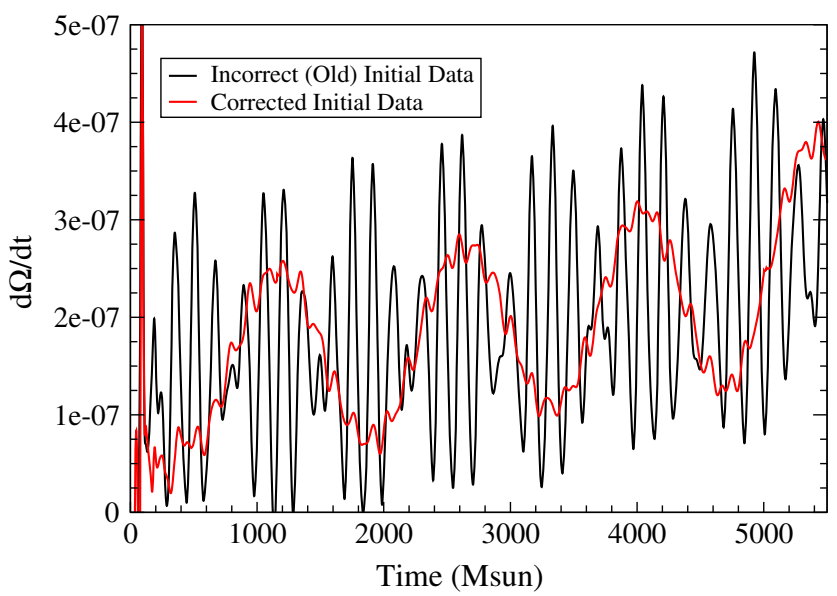

FIG. 2. Derivative of the orbital angular frequency from the $S 0.4 \mathrm{z}$ run and from a new run with the same parameters.

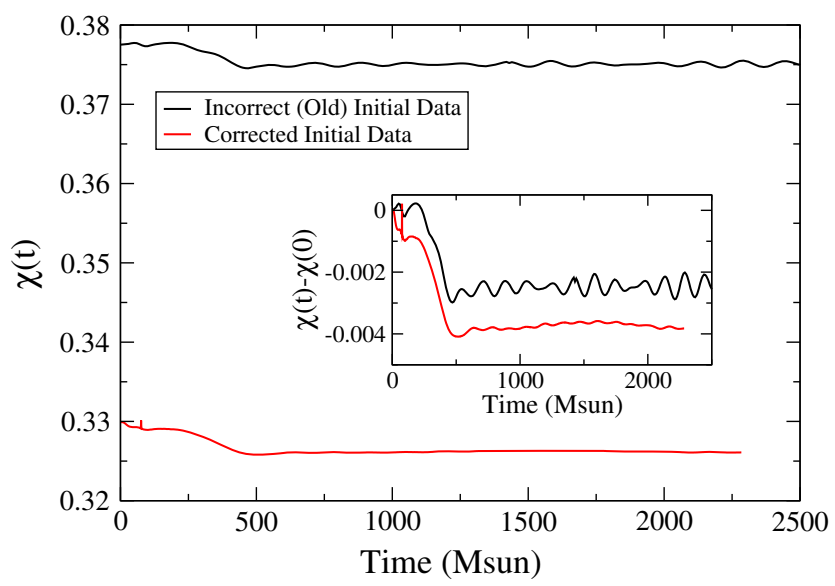

FIG. 3. Dimensionless spin, $\chi=J / M_{\mathrm{ADM}}^{2}$, measured during the evolution of the $\mathrm{S} 0.4 \mathrm{z}-\mathrm{ECC} 3$ run, computed from old and corrected initial data. The inset subtracts the value of the spin at $t=0$ from both curves.

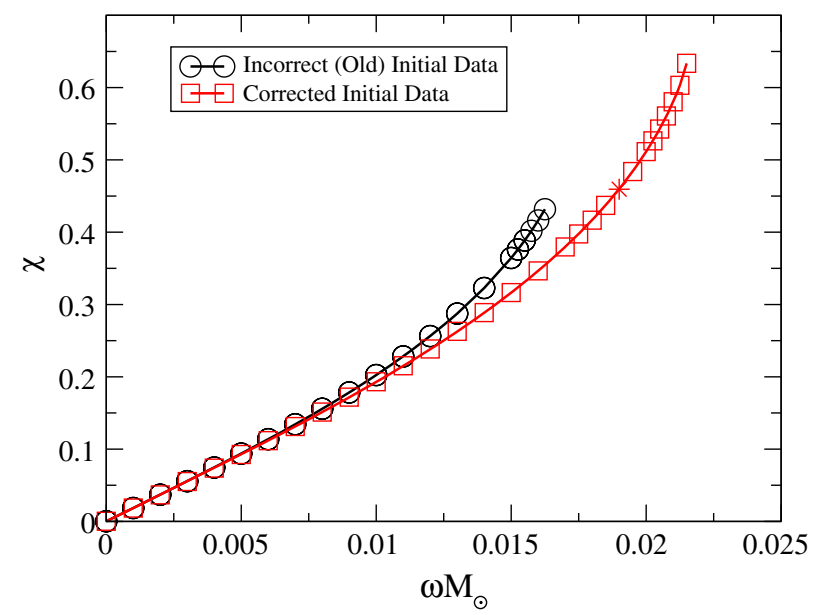

FIG. 4. $\chi$ as a function of $\omega$ in the initial data. The black curve is from Fig. 8 of the original publication, while the red curve is generated with the corrected initial data. The asterisk indicates the configuration we evolve in Fig. 5. 

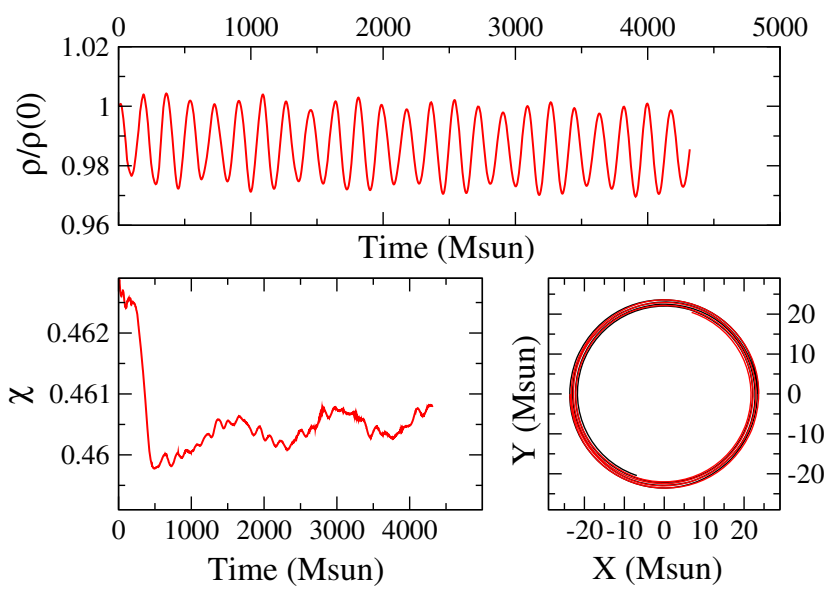

FIG. 5. A snapshot of an evolution with $\omega=0.019$. The top panel shows the normalized density oscillations. The bottom-left panel shows the measured spin of a star. The bottom-right panel shows the orbits of the stars as they inspiral.

magnitude is comparable for both the incorrect and corrected initial data (cf. inset of Fig. 3), although the oscillations present in the data are reduced.

We also find that the corrected code is capable of solving initial data sets for higher values of the NS rotation parameter $\omega$. Figure 3 already showed that at the same rotation parameter $\omega$, the corrected code yields smaller spin. Computing a sequence of initial data sets at different $\omega$, we obtain Fig. 4. For small $\omega$, the $\chi(\omega)$ relation is unchanged, indicating that the low-spin evolution reported in the original article is probably only mildly affected. For large $\omega$, the initial data solver can create ID at spins up to $\chi \sim 0.63$, a factor $\sim 1.4$ larger than the erroneous code. This is, in fact, greater than the breakup spin of $\chi=0.57$ found for $\Gamma=2$ polytropes in Ref. [3].

Being now able to construct ID at larger neutron star spins, we evolve an equal-mass, equal-spin ID set with $\omega=0.019 M_{\odot}^{-1}, \chi=0.46$. In Fig. 5 we present a snapshot of the run, plotting the normalized density oscillations, spin, and the trajectories of the stars. The peak-to-peak density oscillations are now about $2 \%$, higher than in the $\chi \sim 0.33$ evolution, but still much smaller than for the erroneous initial data, despite the larger NS spin.

\section{ACKNOWLEDGMENTS}

We gratefully acknowledge support for this research at CITA from NSERC of Canada, the Canada Research Chairs Program, the Canadian Institute for Advanced Research; at LBNL from NASA through Einstein Postdoctoral Fellowship Grant No. PF4-150122 (F. F.) awarded by the Chandra X-ray Center, which is operated by the Smithsonian Astrophysical Observatory for NASA under Contract No. NAS8-03060; at Caltech from the Sherman Fairchild Foundation and NSF Grants No. PHY-1440083, No. PHY-1404569, No. PHY-1068881, CAREER Grant No. PHY-1151197, TCAN Grant No. AST-1333520, and NASA ATP Grant No. NNX11AC37G; at Cornell from the Sherman Fairchild Foundation and NSF Grants No. PHY-1306125 and No. AST-1333129; and at WSU from NSF Grant No. PHY-1402916. Calculations were performed at the GPC supercomputer at the SciNet HPC Consortium [4]; SciNet is funded by the Canada Foundation for Innovation (CFI) under the auspices of Compute Canada; the government of Ontario; the Ontario Research Fund (ORF) Research Excellence; and the University of Toronto.

[1] W. Tichy, Phys. Rev. D 86, 064024 (2012).

[2] E. Gourgoulhon and S. Bonazzola, Classical Quantum Gravity 11, 443 (1994).

[3] M. Ansorg, A. Kleinwachter, and R. Meinel, Astron. Astrophys. 405, 711 (2003).

[4] C. Loken, D. Gruner, L. Groer, R. Peltier, N. Bunn, M. Craig, T. Henriques, J. Dempsey, C.-H. Yu, J. Chen, L. J. Dursi, J. Chong, S. Northrup, J. Pinto, N. Knecht, and R. V. Zon, J. Phys. Conf. Ser. 256, 012026 (2010). 